\title{
Solutions to BSDEs Driven by Multidimensional Fractional Brownian Motions
}

\author{
Jie Miao ${ }^{1,2}$ and Xu Yang ${ }^{1}$ \\ ${ }^{1}$ School of Mathematics, Shandong University, Jinan, Shandong 250100, China \\ ${ }^{2}$ Mathematics Department, Changji College, Changji, Xinjiang 831100, China \\ Correspondence should be addressed to Jie Miao; miaojie2005_1997@163.com
}

Received 16 December 2014; Accepted 2 March 2015

Academic Editor: P. Balasubramaniam

Copyright (c) 2015 J. Miao and X. Yang. This is an open access article distributed under the Creative Commons Attribution License, which permits unrestricted use, distribution, and reproduction in any medium, provided the original work is properly cited.

We study more general backward stochastic differential equations driven by multidimensional fractional Brownian motions. Introducing the concept of the multidimensional fractional (or quasi-) conditional expectation, we study some of its properties. Using the quasi-conditional expectation and multidimensional fractional Itô formula, we obtain the existence and uniqueness of the solutions to BSDEs driven by multidimensional fractional Brownian motions, where a fixed point principle is employed. Finally, solutions to linear fractional backward stochastic differential equations are investigated.

\section{Introduction}

Increasing attention has been paid to fractional Brownian motion recently, since it can more accurately describe many phenomena in mathematical finance, hydrology, queueing theory, and so on. A centered Gaussian process $\left\{B_{t}^{H}, t \geq\right.$ $0\}$ is called fractional Brownian motion of Hurst parameter $H \in(0,1)$ if it has the covariance function $\mathbb{E}\left[B_{t} B_{s}\right]=$ $(1 / 2)\left(|t|^{2 H}+|s|^{2 H}-|t-s|^{2 H}\right)$. This process was first introduced by Kolmogorov [1] and studied by Mandelbrot and van Ness [2], where a stochastic integral representation in terms of a standard Brownian motion was established. Unfortunately, $\left\{B_{t}^{H}, t \geq 0\right\}$ is neither a semimartingale nor a Markov process, so the powerful tools from the classical theories are not applicable when studying $\left\{B_{t}^{H}, t \geq 0\right\}$. Nevertheless, an efficient stochastic calculus of $\left\{B_{t}^{H}, t \geq 0\right\}$ has been developed in [3-5]. This calculus uses an Itô type integration with respect to $\left\{B_{t}^{H}, t \geq 0\right\}$ and white noise theory. The theory was extended to multiparameter fractional Brownian motion fields $B^{H}(x), x \in \mathbb{R}^{d}$, in [3] and applied to stochastic differential equations driven by such fractional white noise in [6]. In many applications such as mathematical finance, the stochastic calculus of a fractional Brownian motion is needed (see $[7,8])$.
Pardoux and Peng [9] first proved the existence and uniqueness of the solution of general nonlinear BSDEs. Since then, the theory of BSDEs has been extensively studied by many researchers and BSDEs have found applications in many fields, such as finance, risk measure, stochastic control, and so forth. These equations are of the form

$$
y_{t}=\xi+\int_{t}^{T} f\left(s, y_{s}, z_{s}\right) d s+\int_{t}^{T} z_{s} d W_{s}
$$

where $W_{s}$ is a standard Brownian motion and the terminal value $\xi$ and generator $f\left(s, y_{s}, z_{s}\right)$ are given.

In [10] Hu and Peng studied the backward stochastic differential equations driven by a fractional Brownian motion. The equations are as follows:

$$
y_{t}=\xi+\int_{t}^{T} f\left(s, \eta_{s}, y_{s}, z_{s}\right) d s+\int_{t}^{T} z_{s} d B_{s}^{H},
$$

where $\xi=g\left(\eta_{T}\right), g$ is a continuous function, $\eta_{s}=$ $\eta_{0}+b_{s}+\int_{0}^{s} \sigma_{r} d B_{r}^{H}$ with $\eta_{0}, b_{s}$, and $\sigma_{r}$ being deterministic constants or functions, and $B_{r}^{H}$ is a fractional Brownian motion. They introduced the quasi-conditional expectation 
and first discussed its properties. They proved the existence and uniqueness of the solutions to above equations (2). When

$$
f\left(s, \eta_{s}, y_{s}, z_{s}\right)=A\left(s, \eta_{s}\right) y_{s}+B\left(s, \eta_{s}\right) z_{s}+C\left(s, \eta_{s}\right),
$$

the existence of the solutions was proved in [11]. Zhang [12] studied properties of solutions of linear fractional BSDEs using the quasi-conditional expectation and derived the comparison theorem and comonotonic theorem of the solutions.

Maticiuc and Nie [13] improved the result and omitted the assumption in [10]; they proved an existence and uniqueness of the solution of reflected BSDEs driven by fractional Brownian motion. Jańczak-Borkowska [14] added an additional term integral with respect to an increasing process in [13] and proved that kind of equation has a unique solution.

Fei et al. [15] extended the results in [10] to the BSDEs driven by both standard and fractional Brownian motion:

$$
\begin{aligned}
y_{t}= & g\left(\eta_{T}\right)+\int_{t}^{T} f\left(s, \eta_{s}, y_{s}, z_{1, s}, z_{2, s}\right) d s+\int_{t}^{T} z_{1, s} d B_{s} \\
& +\int_{t}^{T} z_{2, s} d B_{s}^{H},
\end{aligned}
$$

where $\eta_{s}=\eta_{0}+b_{s}+\int_{0}^{s} \sigma_{1, r} d B_{r}+\int_{0}^{s} \sigma_{2, r} d B_{r}^{H}$. Using the quasi-conditional expectation, they discussed the existence and uniqueness of solutions to general BSDEs.

In this paper we will deal with the backward stochastic differential equations driven by multidimensional fractional Brownian motions with a more general terminal condition:

$$
\begin{aligned}
y_{t}= & g\left(\eta_{T}\right)+\int_{t}^{T} f\left(s, \eta_{s}, y_{s}, z_{1, s}, \ldots, z_{m, s}\right) d s \\
& +\sum_{j=1}^{m} \int_{t}^{T} z_{j, s} d B_{s}^{H_{j}},
\end{aligned}
$$

where $\eta_{s}=\left(\eta_{1}(s), \ldots, \eta_{n}(s)\right)$ and

$$
\eta_{i}(t)=\eta_{i}(0)+\int_{0}^{t} b_{i}(s) d s+\sum_{j=1}^{m} \int_{0}^{t} \sigma_{i j}(s) d B_{s}^{H_{j}},
$$

where $B_{s}^{H_{j}}, j=1, \ldots, m$, are independent fractional Brownian motions of Hurst parameter $H_{1}, \ldots, H_{m}$ with $H_{j} \in$ $(1 / 2,1)$. We use a fixed point principle to prove the existence and uniqueness of solutions to BSDEs (5).

The rest of the paper is organized as follows. In Section 2, we recall some basic results of multidimensional fractional Brownian motion. Section 3 is concerned with the multidimensional fractional (or quasi-) conditional expectation. In Section 4, existence and uniqueness of the solutions to the general fractional BSDEs are proved. Solutions of the linear fractional BSDEs are discussed in Section 5. The conclusions are set out in Section 6.

\section{Preliminaries}

In this section, we first recall some basic results of multidimensional fractional Brownian motions.
Let $\left\{B_{t}^{H_{i}}, t \in[0, T]\right\}, i=1, \ldots, n$, be independent fractional Brownian motions of Hurst parameters $H_{1}, \ldots, H_{n}$ with $H_{i} \in(1 / 2,1)$ on a filtered probability space $\left(\Omega, \mathscr{F},\left\{\mathscr{F}_{t}, t \in[0, T]\right\}, \mathbb{P}\right)$, where the filtration $\left\{\mathscr{F}_{t}, t \in\right.$ $[0, T]\}$ is the $\sigma$-algebra generated by $\left\{B_{t}^{H_{i}}, t \in[0, T], i=\right.$ $1, \ldots, n\}$. The process $\left\{B_{t}^{H_{i}}, t \in[0, T], i=1, \ldots, n\right\}$ satisfies

$$
\begin{gathered}
\mathbb{E}\left[B_{t}^{H_{i}}\right]=0, \\
\mathbb{E}\left[B_{t}^{H_{i}} B_{s}^{H_{j}}\right]=\frac{1}{2}\left(|t|^{2 H_{i}}+|s|^{2 H_{i}}-|t-s|^{2 H_{i}}\right) \delta_{i j} ; \\
1 \leq i, j \leq n, \quad s, t \in[0, T],
\end{gathered}
$$

where

$$
\delta_{i j}= \begin{cases}0, & i \neq j \\ 1, & i=j .\end{cases}
$$

Let $f$ and $g$ be two continuous functions on $[0, T]$. Define

$$
\langle f, g\rangle_{i, t}=\int_{0}^{t} \int_{0}^{t} \phi_{i}(u, v) f(u) g(v) d u d v
$$

where $\phi_{i}(u, v)=H_{i}\left(2 H_{i}-1\right)|u-v|^{2 H_{i}-2}$. If $f=g$, then we denote

$$
\|f\|_{i, t}^{2}=\int_{0}^{t} \int_{0}^{t} \phi_{i}(u, v) f(u) f(v) d u d v .
$$

If $F: \Omega \rightarrow \mathbb{R}$ is a given function, let $\mathbb{D}_{t}^{H_{i}} F$ of $F$ at time $t$ be the Malliavin $\phi_{i}$-derivative; then

$$
\mathbb{D}_{t}^{H_{i}} F=\int_{0}^{t} \phi_{i}(s, t) D_{s}^{H_{i}} F d s
$$

where $D_{s}^{H_{i}} F$ is Malliavin derivative of $F$ at time $s$. More detailed study about Malliavin derivative is in [16].

We now give the multidimensional fractional Itô formula.

Lemma 1 (multidimensional fractional Itô formula [3, 17]). For $i, k=1, \ldots, n, j=1, \ldots, m$, let $\sigma_{i j} \in L^{2}([0, T])$ be deterministic functions and let $\left\langle\sigma_{i k}, \sigma_{j k}\right\rangle_{k, t}$ be continuously differentiable as functions of $t \in[0, T]$. Denote $\xi(t)=$ $\left(\xi_{1}(t), \ldots, \xi_{n}(t)\right)$ :

$$
\begin{array}{r}
\xi_{i}(t)=\xi_{i}(0)+\int_{0}^{t} b_{i}(s) d s+\sum_{j=1}^{m} \int_{0}^{t} \sigma_{i j}(s) d B_{s}^{H_{j}}, \\
0 \leq t \leq T,
\end{array}
$$

where $\xi(0)=\left(\xi_{1}(0), \ldots, \xi_{n}(0)\right)$ is a constant vector and $b_{i}(s), i=1, \ldots, n$, are continuous deterministic functions with $\int_{0}^{T}\left|b_{i}(s)\right| d s<\infty$. Let $F(t, x)$ be continuously differentiable 
with respect to $t$ and twice continuously differentiable with respect to $x$. Then

$$
\begin{aligned}
F( & t, \xi(t)) \\
= & F(0, \xi(0))+\int_{0}^{t} \frac{\partial F}{\partial s}(s, \xi(s)) d s \\
& +\sum_{i=1}^{n} \int_{0}^{t} \frac{\partial F}{\partial x_{i}}(s, \xi(s)) d \xi_{i}(s) \\
& +\sum_{i, j=1}^{n} \int_{0}^{t} \frac{\partial^{2} F}{\partial x_{i} x_{j}}(s, \xi(s)) \sum_{k=1}^{m} \sigma_{i k}(s) \mathbb{D}_{s}^{H_{k}}\left(\xi_{j}(s)\right) d s . \\
\text { Since } & \mathbb{D}_{s}^{H_{k}}\left(\xi_{j}(s)\right)=\int_{0}^{s} \phi_{k}(s, v) \sigma_{j k}(v) d v, \text { we obtain } \\
F(t, & \xi(t)) \\
= & F(0, \xi(0))+\int_{0}^{t} \frac{\partial F}{\partial s}(s, \xi(s)) d s \\
& +\sum_{i=1}^{n} \int_{0}^{t} \frac{\partial F}{\partial x_{i}}(s, \xi(s)) b_{i}(s) d s \\
& +\sum_{i=1}^{n} \sum_{j=1}^{m} \int_{0}^{t} \frac{\partial F}{\partial x_{i}}(s, \xi(s)) \sigma_{i j}(s) d B_{s}^{H_{j}} \\
& +\sum_{i, j=1}^{n} \int_{0}^{t} \frac{\partial^{2} F}{\partial x_{i} x_{j}}(s, \xi(s)) \sum_{k=1}^{m}\left[\frac{d}{d s}\left\langle\sigma_{i k}, \sigma_{j k}\right\rangle_{k, s}\right] d s,
\end{aligned}
$$

where

$$
\begin{aligned}
& {\left[\frac{d}{d s}\left\langle\sigma_{i k}, \sigma_{j k}\right\rangle_{k, s}\right]} \\
& \quad=2 H_{k}\left(2 H_{k}-1\right) \sigma_{i k}(s) \int_{0}^{s}(s-v)^{2 H_{k}-2} \sigma_{j k}(v) d v .
\end{aligned}
$$

By the two-dimensional fractional Itô formula, we can obtain the following chain rule.

Lemma 2 (fractional Itô chain rule $[3,17]$ ). Let $b_{1}(s), b_{2}(s)$, $\sigma_{i 1}(s), \sigma_{i 2}(s), 0 \leq s \leq T, i=1, \ldots, n$, be real-valued stochastic processes such that

$$
\begin{array}{r}
\mathbb{E}\left\{\int_{0}^{T}\left|b_{j}(s)\right|^{2} d s+\sum_{i=1}^{n} \int_{0}^{T}\left|\sigma_{i j}(s)\right|^{2} d s\right\} \\
j=\infty \\
j=1,2 .
\end{array}
$$

Assume also that $\mathbb{D}_{t}^{H_{i}} b_{j}(s)$ and $\mathbb{D}_{t}^{H_{i}} \sigma_{i j}(s), i=1, \ldots, n, j=1,2$, are continuously differentiable with respect to $(s, t) \in[0, T]^{2}$ for almost all $\omega \in \Omega$. Suppose that $\mathbb{E}\left\{\int_{0}^{T} \int_{0}^{T}\left|\mathbb{D}_{t}^{H_{i}} b_{j}(s)\right|^{2} d s d t\right\}<\infty$ and $\mathbb{E}\left\{\int_{0}^{T} \int_{0}^{T}\left|\mathbb{D}_{t}^{H_{i}} \sigma_{i j}(s)\right|^{2} d s d t\right\}<\infty, i=1, \ldots, n, j=1,2$. Denote

$$
\begin{array}{r}
X_{j}(t)=X_{j}(0)+\int_{0}^{t} b_{j}(s) d s+\sum_{i=1}^{n} \int_{0}^{t} \sigma_{i j}(s) d B_{s}^{H_{i}}, \\
t \in[0, T], \quad j=1,2 .
\end{array}
$$

Then

$$
\begin{aligned}
X_{1}(t) X_{2}(t)= & X_{1}(0) X_{2}(0)+\int_{0}^{t} X_{1}(s) d X_{2}(s) \\
& +\int_{0}^{t} X_{2}(s) d X_{1}(s) \\
& +\sum_{i=1}^{n} \int_{0}^{t} \mathbb{D}_{s}^{H_{i}} X_{1}(s) \sigma_{i 2}(s) d s \\
& +\sum_{i=1}^{n} \int_{0}^{t} \mathbb{D}_{s}^{H_{i}} X_{2}(s) \sigma_{i 1}(s) d s,
\end{aligned}
$$

which can be written as a differential form:

$$
\begin{aligned}
d & \left.X_{1}(t) X_{2}(t)\right) \\
= & X_{1}(t) d X_{2}(t)+X_{2}(t) d X_{1}(t) \\
& +\left[\sum_{i=1}^{n} \mathbb{D}_{t}^{H_{i}} X_{1}(t) \sigma_{i 2}(t)+\sum_{i=1}^{n} \mathbb{D}_{t}^{H_{i}} X_{2}(t) \sigma_{i 1}(t)\right] d t .
\end{aligned}
$$

We know that fractional Brownian motion and Brownian motion have the following relationship:

$$
B_{t}^{H_{i}}=\int_{0}^{t} K_{H_{i}}(t, s) d W_{s},
$$

where $\left(W_{s}, s \geq 0\right)$ is a standard Brownian motion and

$$
\begin{aligned}
& K_{H_{i}}(t, s) \\
&=C_{H_{i}}\left[\left(\frac{t}{s}\right)^{H_{i}-1 / 2}(t-s)^{H_{i}-1 / 2}\right. \\
& \\
&\left.-\left(H_{i}-\frac{1}{2}\right) s^{1 / 2-H_{i}} \int_{0}^{t} u^{H_{i}-3 / 2}(u-s)^{H_{i}-1 / 2} d u\right],
\end{aligned}
$$

with $C_{H_{i}}$ being a constant such that $\int_{0}^{1} K_{H_{i}}^{2}(1, s) d s=1$. With this $K_{H_{i}}(t, s)$ we associate an operator

$$
\mathscr{K}_{H_{i}}(t)=\int_{0}^{t} K_{H_{i}}(t, s) h(s) d s, \quad 0 \leq t<\infty .
$$

This operator has an inverse operator which can be expressed as

$$
\mathscr{K}_{H_{i}}^{-1}(t)=s^{H_{i}-1 / 2} D_{0+}^{H_{i}-1 / 2}\left(r^{1 / 2-H_{i}} h^{\prime}(r)\right)(t),
$$

where (see [6])

$$
\begin{aligned}
& D_{0+}^{H_{i}-1 / 2} f(t) \\
& =\frac{1}{\Gamma\left(3 / 2-H_{i}\right)}\left(\frac{f(t)}{t^{H_{i}-1 / 2}}+\left(H_{i}-\frac{1}{2}\right) \int_{0}^{t} \frac{f(t)-f(s)}{(t-s)^{H_{i}+1 / 2}} d s\right) .
\end{aligned}
$$

Let us consider the translation

$$
\widetilde{B}_{t}^{H_{i}}=B_{t}^{H_{i}}-\int_{0}^{t} b_{s} d s,
$$


where $b_{s}$ is a continuous and adapted process; then

$$
\begin{aligned}
\widetilde{B}_{t}^{H_{i}}= & \int_{0}^{t} K_{H_{i}}(t, s) d W_{s} \\
& -\int_{0}^{t} K_{H_{i}}(t, s)\left(K_{H_{i}}^{-1} \int_{0} b_{r} d r\right)(s) d s \\
= & \int_{0}^{t} K_{H_{i}}(t, s) d \widetilde{W}_{s},
\end{aligned}
$$

where

$$
\widetilde{W}_{t}=W_{t}-\int_{0}^{t}\left(K_{H_{i}}^{-1} \int_{0} b_{r} d r\right)(s) d s
$$

By the Novikov condition, if

$$
\mathbb{E}\left[\exp \left\{\frac{1}{2} \int_{0}^{T}\left(K_{H_{i}}^{-1} \int_{0} b_{r} d r\right)(s) d s\right\}\right]<\infty
$$

then

$$
\begin{aligned}
\exp \left\{\int_{0}^{t}\left(K_{H_{i}}^{-1} \int_{0}^{\cdot} b_{r} d r\right)(s) d W_{s}\right. & \left.\quad \frac{1}{2} \int_{0}^{t}\left(K_{H_{i}}^{-1} \int_{0} b_{r} d r\right)^{2}(s) d s\right\}, \quad 0 \leq t \leq T,
\end{aligned}
$$

which is an exponential martingale. Define $\widetilde{P}$ by the following:

$$
\begin{aligned}
\frac{d \widetilde{P}}{d P}=\exp \left\{\int_{0}^{t}\left(K_{H_{i}}^{-1} \int_{0} b_{r} d r\right)(s) d W_{s}\right. \\
\left.\quad-\frac{1}{2} \int_{0}^{t}\left(K_{H_{i}}^{-1} \int_{0} b_{r} d r\right)^{2}(s) d s\right\} .
\end{aligned}
$$

Then, under the probability measure $\widetilde{P}, \widetilde{B}_{t}^{H_{i}}$ is a new fractional Brownian motion.

\section{Quasi-Conditional Expectation}

In this section, we introduce multidimensional fractional (or quasi-) conditional expectation.

Let $\|f\|_{t}=\left(\iint_{0}^{t} \phi_{i}(u, v) f(u) f(v) d u d v\right)^{1 / 2} \cdot \mathscr{H}_{t}^{i}$ denotes the completion space of continuous functions under the Hilbert norm $\|\cdot\|_{t}$. Let $\mathscr{H}_{t}^{i \otimes n}$ denote the set of all real symmetric functions $f_{n}$ of $n$ variables on $[0, t]$ such that

$$
\begin{aligned}
\sum_{n=0}^{\infty} n ! & \int_{[0, t]^{2 n}}\left|f_{n}\left(s_{1}, \ldots, s_{n}\right)\right|\left|f_{n}\left(t_{1}, \ldots, t_{n}\right)\right| \\
\cdot & \prod_{j=1}^{n} \phi_{i}\left(s_{j}, t_{j}\right) d s_{1} \cdots d s_{n} d t_{1} \cdots d t_{n}<\infty .
\end{aligned}
$$

Let $\widehat{L}^{2}(\Omega, \mathscr{F}, P)$ denote the set of $F \in L^{2}(\Omega, \mathscr{F}, P)$ such that $F$ has the following chaos expansion:

$$
\begin{aligned}
F(\omega) & \\
& =\sum_{i=1}^{m} \sum_{n=0}^{\infty} I_{n}^{H_{i}}\left(f_{n}\right) \\
& =\sum_{i=1}^{m} \sum_{n=0}^{\infty} \int_{[0, T]^{n}} f_{n}\left(s_{1}, \ldots, s_{n}\right) d B_{s_{1}}^{H_{i}} \cdots d B_{s_{n}}^{H_{i}} \\
& =\sum_{i=1}^{m} \sum_{n=0}^{\infty} n ! \int_{0}^{T} \cdots \int_{0}^{s_{2}} f_{n}\left(s_{1}, \ldots, s_{n}\right) d B_{s_{1}}^{H_{i}} \cdots d B_{s_{n}}^{H_{i}},
\end{aligned}
$$

where $f_{n} \in \mathscr{H}_{t}^{i \otimes n}$.

Now we introduce the quasi-conditional expectation for multidimensional fractional Brownian motion, which is important in solving backward stochastic differential equations.

Definition 3. The quasi-conditional expectation of some random variable $F \in \widehat{L}^{2}(\Omega, \mathscr{F}, P)$ relative to a fractional Brownian motion with Hurst parameter $H_{i}$ is defined by

$$
\widehat{\mathbb{E}}_{P}^{H_{i}}\left[F \mid \mathscr{F}_{t}\right]=\sum_{n=0}^{\infty} I_{n}^{H_{i}}\left(f_{n} I_{[0, t]}\right),
$$

where $I_{[0, t]}\left(t_{1}, \ldots, t_{n}\right)=I_{[0, t]}\left(t_{1}\right) \cdots I_{[0, t]}\left(t_{n}\right)$.

Let $G \in \widehat{L}^{2}(\Omega, \mathscr{F}, P)$, since $B_{t}^{H_{i}}, i=1, \ldots, m$, are independent fractional Brownian motions; by Definition 3, we have

$$
\widehat{\mathbb{E}}_{P}^{H}\left[G \mid \mathscr{F}_{t}\right]=\hat{\mathbb{E}}_{P}^{H_{1}}\left[\cdots \hat{\mathbb{E}}_{P}^{H_{m}}\left[G \mid \mathscr{F}_{t}\right] \cdots \mid \mathscr{F}_{t}\right] .
$$

Lemma 4. If $f_{s}$ is the real stochastic processes such that

$$
\begin{gathered}
\mathbb{E}\left[\int_{t}^{T} \int_{t}^{T} \phi_{i}(u, v)|f(u)||f(v)| d u d v\right. \\
\left.+\int_{t}^{T} \int_{t}^{T}\left|\mathbb{D}_{u}^{H_{i}} f(v)\right|^{2} d u d v\right]<\infty,
\end{gathered}
$$

and $\int_{t}^{T} f(s) d B_{s}^{H_{i}} \in \widehat{L}^{2}(\Omega, \mathscr{F}, P)$, then

$$
\widehat{\mathbb{E}}_{P}^{H_{i}}\left[\int_{t}^{T} f(s) d B_{s}^{H_{i}} \mid \mathscr{F}_{t}\right]=0 .
$$

We introduce a function

$$
P_{t} f(x)=(2 \pi t)^{-n / 2} \int_{\mathbb{R}^{n}} e^{-(x-y)^{2} / 2 t} f(y) d y .
$$

Let $W_{t}$ be a standard Brownian motion started at 0 and let $\beta$ be a $n \times n$-matrix; one defines an $\mathbb{R}^{n}$-valued Markov process $X$ by stipulation that if $X_{0}=x$ a.s., then $X_{t}=x+\beta W_{t}$, where $W_{t}$ is $n$-dimensional Brownian motion. If $f$ is continuous, then we have

$$
\frac{\partial}{\partial t} P_{t} f(x)=\frac{1}{2} \sum_{i, j=1}^{n} \gamma_{i j} \frac{\partial^{2}}{\partial x_{i} \partial x_{j}} P_{t} f(x),
$$

where $\gamma=\beta \beta^{t}$, with $\beta^{t}$ being the transpose of $\beta$ (see [18]). 
Theorem 5. Let $\eta_{i}(t)=\sum_{j=1}^{m} \int_{0}^{t} \sigma_{i j}(s) d B_{s}^{H_{j}}, i=1, \ldots, n$, $F=f\left(\eta_{T}\right)$, and $G=g\left(\eta_{T}\right)$, where $\eta_{T}=\left(\eta_{1}(T), \ldots, \eta_{n}(T)\right)$. For $i, j=1, \ldots, n, k=1, \ldots, m$, and $s \in[0, T]$, assume that $\sigma_{i j}(s), i=1, \ldots, n, j=1, \ldots, m$, are measurable and $\left[(d / d s)\left\langle\sigma_{i k}, \sigma_{j k}\right\rangle_{k, s}\right]>0$. Let $A_{t}=\left(A_{i j}(t)\right)$ be $n \times n$-matrix, where

$$
A_{i j}(t)=\sum_{k=1}^{m}\left\langle\sigma_{i k}, \sigma_{j k}\right\rangle_{k, t} .
$$

If $F \geq G$ almost surely, then

$$
\widehat{\mathbb{E}}_{P}^{H}\left[F \mid \mathscr{F}_{t}\right] \geq \widehat{\mathbb{E}}_{P}^{H}\left[G \mid \mathscr{F}_{t}\right] .
$$

We also have

$$
\widehat{\mathbb{E}}_{P}^{H}\left[F \mid \mathscr{F}_{t}\right]=P_{A_{T}-A_{t}} f\left(\eta_{t}\right) .
$$

Proof. It suffices to show the case $G=0$. Applying Lemma 1 to $P_{A_{T}-A_{t}} f\left(\eta_{t}\right)$, we have

$$
\begin{aligned}
& f\left(\eta_{T}\right)=P_{A_{T}} f\left(\eta_{0}\right)+\int_{0}^{T} \frac{\partial}{\partial s} P_{A_{T}-A_{s}} f\left(\eta_{s}\right) d s \\
& +\sum_{i=1}^{n} \int_{0}^{T} \frac{\partial}{\partial x_{i}} P_{A_{T}-A_{s}} f\left(\eta_{s}\right)\left[\sum_{j=1}^{m} \sigma_{i j}(s) d B_{s}^{H_{j}}\right] \\
& +\sum_{i, j=n}^{m} \int_{0}^{T} \frac{\partial^{2}}{\partial x_{i} x_{j}} P_{A_{T}-A_{s}}\left(\eta_{s}\right) \\
& \quad \cdot \sum_{k=1}^{m}\left[\frac{d}{d s}\left\langle\sigma_{i k}, \sigma_{j k}\right\rangle_{k, s}\right] d s .
\end{aligned}
$$

By (38) and the chain rule of composite function, we deduce

$$
\begin{aligned}
& \frac{\partial}{\partial s} P_{A_{T}-A_{s}} f\left(\eta_{s}\right) \\
& \quad=-\sum_{i, j=n}^{m} \frac{\partial^{2}}{\partial x_{i} x_{j}} P_{A_{T}-A_{s}}\left(\eta_{s}\right) \sum_{k=1}^{m}\left[\frac{d}{d s}\left\langle\sigma_{i k}, \sigma_{j k}\right\rangle_{k, s}\right] .
\end{aligned}
$$

By (42) and (43), we obtain

$$
\begin{aligned}
& f\left(\eta_{T}\right) \\
& \quad=P_{A_{T}} f\left(\eta_{0}\right) \\
& \quad+\sum_{i=1}^{n} \int_{0}^{T} \frac{\partial}{\partial x_{i}} P_{A_{T}-A_{s}} f\left(\eta_{s}\right)\left[\sum_{j=1}^{m} \sigma_{i j}(s) d B_{s}^{H_{j}}\right] .
\end{aligned}
$$

Taking the quasi-conditional expectation both sides of (44), we have

$$
\begin{aligned}
\widehat{\mathbb{E}}_{P}^{H}\left[F \mid \mathscr{F}_{t}\right] & \\
= & P_{A_{T}} f\left(\eta_{0}\right) \\
& \quad+\sum_{i=1}^{n} \int_{0}^{t} \frac{\partial}{\partial x_{i}} P_{A_{T}-A_{s}} f\left(\eta_{s}\right)\left[\sum_{j=1}^{m} \sigma_{i j}(s) d B_{s}^{H_{j}}\right] .
\end{aligned}
$$

But

$$
\begin{aligned}
f\left(\eta_{t}\right)= & P_{A_{t}} f\left(\eta_{0}\right) \\
& +\sum_{i=1}^{n} \int_{0}^{t} \frac{\partial}{\partial x_{i}} P_{A_{t}-A_{s}} f\left(\eta_{s}\right)\left[\sum_{j=1}^{m} \sigma_{i j}(s) d B_{s}^{H_{j}}\right],
\end{aligned}
$$

from the semigroup property of $P_{A_{t}}$; we have

$$
P_{A_{T}-A_{s}} f(x)=P_{A_{T}-A_{t}} P_{A_{t}-A_{s}} f(x),
$$

and it is easy to check that

$$
\frac{\partial}{\partial x} P_{A_{T}-A_{s}} f(x)=P_{A_{T}-A_{t}} \frac{\partial}{\partial x} P_{A_{t}-A_{s}} f(x) .
$$

Hence

$$
\begin{aligned}
\widehat{\mathbb{E}}_{P}^{H} & {\left[F \mid \mathscr{F}_{t}\right] } \\
= & P_{A_{T}-A_{t}} P_{A_{t}} f\left(\eta_{0}\right) \\
& \quad+\sum_{i=1}^{n} P_{A_{T}-A_{t}} \int_{0}^{t} \frac{\partial}{\partial x_{i}} P_{A_{t}-A_{s}} f\left(\eta_{s}\right)\left[\sum_{j=1}^{m} \sigma_{i j}(s) d B_{s}^{H_{j}}\right] \\
= & P_{A_{T}-A_{t}} f\left(\eta_{t}\right) .
\end{aligned}
$$

The assertion is completed.

\section{Backward Stochastic Differential Equations Driven by Multidimensional Fractional Brownian Motions}

In this section we will consider a backward stochastic differential equation driven by multidimensional fractional Brownian motions.

Denote $x_{t}=\left(x_{1}(t), x_{2}(t), \ldots, x_{n}(t)\right)$, where

$$
\begin{array}{r}
x_{i}(t)=x_{i}(0)+\int_{0}^{t} b_{i}(s) d s+\sum_{j=1}^{m} \int_{0}^{t} \sigma_{i j}(s) d B_{s}^{H_{j}}, \\
i=1, \ldots, n,
\end{array}
$$

and $B_{s}^{H_{j}}, \ldots, B_{s}^{H_{m}}$ are independent fractional Brownian motions of Hurst parameters $H_{1}, \ldots, H_{m}$.

Assume that

$\left(H_{1}\right) x_{i}(0), i=1, \ldots, n$, are given constants.

$\left(H_{2}\right)$ For $i=1, \ldots, n, b_{i}:[0, T] \rightarrow \mathbb{R}$ are continuous deterministic functions.

$\left(H_{3}\right)$ For $i, j=1, \ldots, n, k=1, \ldots, m, \sigma_{i k}:[0, T] \rightarrow \mathbb{R}$ are continuous deterministic functions, and $\left[(d / d t)\left\langle\sigma_{i k}, \sigma_{j k}\right\rangle_{k, t}\right]>0$, where $\left[(d / d t)\left\langle\sigma_{i k}, \sigma_{j k}\right\rangle_{k, t}\right]=$ $2 H_{k}\left(2 H_{k}-1\right) \sigma_{i k}(t) \int_{0}^{t}(t-s)^{\left(2 H_{i}-2\right)} \sigma_{j k}(s) d s$.

$\left(H_{4}\right)$ For $i=1, \ldots, n, j=1, \ldots, m$,

$$
\inf _{0 \leq t \leq T} \frac{\widehat{\sigma}_{i j}(t)}{\sigma_{i j}(t)} \geq c_{0}>0,
$$

where $\widehat{\sigma}_{i j}(t)=\int_{0}^{t} \phi_{j}(t, r) \sigma_{i j}(r) d r$. 
Now we consider the following backward stochastic differential equation:

$$
\begin{aligned}
d y_{t} & =-f\left(t, x_{t}, y_{t}, z_{t}\right) d t-\sum_{j=1}^{m} z_{j, t} d B_{t}^{H_{j}}, \\
y_{T} & =g\left(x_{T}\right) .
\end{aligned}
$$

That is,

$$
\begin{array}{r}
y_{t}=g\left(x_{T}\right)+\int_{t}^{T} f\left(s, x_{s}, y_{s}, z_{s}\right) d s+\sum_{j=1}^{m} \int_{t}^{T} z_{j, s} d B_{s}^{H_{j}}, \\
0 \leq t \leq T,
\end{array}
$$

where $z_{s}=\left(z_{1, s}, \ldots, z_{m, s}\right)$.

We further assume that

$\left(H_{5}\right) g(x)$ is a continuously differentiable function with respect to $x$ and satisfies polynomial growth.

$\left(H_{6}\right) f(t, x, y, z)$ is a continuous function with respect to $t$ and twice continuously differentiable with respect to $x, y$, and $z$, so there exists a constant $L>0$, such that, for all $t \in[0, T], x \in \mathbb{R}, y, y^{\prime} \in \mathbb{R}$, and $z, z^{\prime} \in \mathbb{R}^{m}$, we have

$$
\begin{aligned}
& \left|f(t, x, y, z)-f\left(t, x, y^{\prime}, z^{\prime}\right)\right| \\
& \quad \leq L\left(\left|y-y^{\prime}\right|+\left|z-z^{\prime}\right|\right) .
\end{aligned}
$$

The partial differential equation associated with above equation (52) is

$$
\begin{aligned}
& u_{t} \\
& =-\frac{1}{2} \sum_{i, j=1}^{n} \sum_{k=1}^{m}\left[\frac{d}{d s}\left\langle\sigma_{i k}, \sigma_{j k}\right\rangle_{k, s}\right] u_{x_{i} x_{j}}-\sum_{i=1}^{n} b_{i}(t) u_{x_{i}} \\
& -f\left(t, x, u,-\sum_{i=1}^{n} \sigma_{i 1}(t) u_{x_{i}}, \ldots,-\sum_{i=1}^{n} \sigma_{i m}(t) u_{x_{i}}\right), \\
& u(T, x)=g(x) . \\
& \left(y_{t}, z_{1, t}, \ldots, z_{m, t}\right) \\
& =\left(u\left(t, x_{t}\right),-\sum_{i=1}^{n} \sigma_{i 1}(t) u_{x_{i}}\left(t, x_{t}\right), \ldots,-\sum_{i=1}^{n} \sigma_{i m}(t) u_{x_{i}}\left(t, x_{t}\right)\right) .
\end{aligned}
$$

Proof. From Itô formula (14), we get

$$
\begin{aligned}
u( & \left.t, x_{t}\right) \\
= & u\left(0, x_{0}\right)+\int_{0}^{t} \frac{\partial u}{\partial s}\left(s, x_{s}\right) d s \\
& +\sum_{i=1}^{n} \int_{0}^{t} \frac{\partial u}{\partial x_{i}}\left(s, x_{s}\right) b_{i}(s) d s \\
& +\sum_{i=1}^{n} \sum_{j=1}^{m} \int_{0}^{t} \frac{\partial u}{\partial x_{i}}\left(s, x_{s}\right) \sigma_{i j}(s) d B_{s}^{H_{j}} \\
& +\int_{0}^{t} \sum_{i, j=1}^{n} \frac{1}{2} \frac{\partial^{2} u}{\partial x_{i} x_{j}}\left(s, x_{s}\right) \sum_{k=1}^{m}\left[\frac{d}{d s}\left\langle\sigma_{i k}, \sigma_{j k}\right\rangle_{k, s}\right] d s \\
= & u\left(0, x_{0}\right) \\
& +\int_{0}^{t}\left\{\frac{\partial u}{\partial s}\left(s, x_{s}\right)+\sum_{i=1}^{n} \frac{\partial u}{\partial x_{i}}\left(s, x_{s}\right) b_{i}(s)\right. \\
& +\sum_{j=1}^{m} \sum_{i=1}^{n} \int_{0}^{t} \frac{\partial u}{\partial x_{i}}\left(s, x_{s}\right) \sigma_{i j}(s) d B_{s}^{H_{j}} \cdot \\
& \left.+\frac{1}{2} \sum_{i, j=1}^{n} \frac{\partial^{2} u}{\partial x_{i} x_{j}}\left(s, x_{s}\right) \sum_{k=1}^{m}\left[\frac{d}{d s}\left\langle\sigma_{i k}, \sigma_{j k}\right\rangle_{k, s}\right]\right\} d s
\end{aligned}
$$

Since $u(t, x)$ satisfies (55), we deduce

$$
\begin{aligned}
& u\left(t, x_{t}\right) \\
& =g\left(x_{T}\right) \\
& \quad+\int_{t}^{T} f\left(s, x, u,-\sum_{i=1}^{n} \sigma_{i 1}(s) u_{x_{i}}, \ldots,-\sum_{i=1}^{n} \sigma_{i m}(s) u_{x_{i}}\right) d s \\
& \quad-\sum_{j=1}^{m} \sum_{i=1}^{n} \int_{t}^{T} \sigma_{i j}(s) u_{x_{i}} d B_{s}^{H_{j}} .
\end{aligned}
$$

From (58), we get

$$
\begin{gathered}
\left(y_{t}, z_{1, t}, \ldots, z_{m, t}\right)=\left(u\left(t, x_{t}\right),-\sum_{i=1}^{n} \sigma_{i 1}(t) u_{x_{i}}\left(t, x_{t}\right), \ldots,\right. \\
\left.\quad-\sum_{i=1}^{n} \sigma_{i m}(t) u_{x_{i}}\left(t, x_{t}\right)\right)
\end{gathered}
$$

which satisfies BSDE (52), and the proof is complete. 
Lemma 7. Let $a(s, x), \theta_{j}(s, x), j=1, \ldots, m$, be continuous with respect to $t$ and continuously differentiable with respect to $x$ and let them be of polynomial growth. Assume that $\left(\mathrm{H}_{2}\right)-$ $\left(\mathrm{H}_{3}\right)$, if

$$
\int_{0}^{t} a\left(s, x_{s}\right) d s+\sum_{j=1}^{m} \int_{0}^{t} \theta_{j}\left(s, x_{s}\right) d B_{s}^{H_{j}}=0
$$

$\forall t \in[0, T]$,

then

$$
a(s, x)=\theta_{1}(s, x)=\cdots=\theta_{m}(s, x)=0 .
$$

Proof. Similar to the proof of Lemma 3.2 in [19], we can obtain Lemma 7.

Theorem 8. Assume that $\left(\mathrm{H}_{3}\right)$ and let (52) have a solution of the form $\left(y_{t}=u\left(t, x_{t}\right), z_{1, t}=v_{1}\left(s, x_{s}\right), \ldots, z_{m, t}=v_{m}\left(s, x_{s}\right)\right)$. Then

$$
\begin{gathered}
v_{1}\left(s, x_{s}\right)=-\sum_{i=1}^{n} \sigma_{i 1}(t) u_{x_{i}}\left(t, x_{t}\right) \\
\vdots \\
v_{m}\left(s, x_{s}\right)=-\sum_{i=1}^{n} \sigma_{i m}(t) u_{x_{i}}\left(t, x_{t}\right) .
\end{gathered}
$$

Proof. From the proof of Theorem 6, we know

$$
\begin{aligned}
& u\left(t, x_{t}\right) \\
& =g\left(x_{T}\right) \\
& \quad-\int_{t}^{T}\left\{\frac{\partial u}{\partial s}\left(s, x_{s}\right)+\sum_{i=1}^{n} \frac{\partial u}{\partial x_{i}}\left(s, x_{s}\right) b_{i}(s)\right. \\
& \left.\quad+\frac{1}{2} \sum_{i, j=1}^{n} \frac{\partial^{2} u}{\partial x_{i} x_{j}}\left(s, x_{s}\right) \sum_{k=1}^{m}\left[\frac{d}{d s}\left\langle\sigma_{i k}, \sigma_{j k}\right\rangle_{s}\right]\right\} d s \\
& -\sum_{j=1}^{m} \sum_{i=1}^{n} \int_{t}^{T} \frac{\partial u}{\partial x_{i}}\left(s, x_{s}\right) \sigma_{i j}(s) d B_{s}^{H_{j}} .
\end{aligned}
$$

Furthermore, we deduce

$$
\begin{aligned}
& -\int_{t}^{T}\left\{\frac{\partial u}{\partial s}\left(s, x_{s}\right)+\sum_{i=1}^{n} \frac{\partial u}{\partial x_{i}}\left(s, x_{s}\right) b_{i}(s)\right. \\
& \left.\quad+\frac{1}{2} \sum_{i, j=1}^{n} \frac{\partial^{2} u}{\partial x_{i} x_{j}}\left(s, x_{s}\right) \sum_{k=1}^{m}\left[\frac{d}{d s}\left\langle\sigma_{i k}, \sigma_{j k}\right\rangle_{k, s}\right]\right\} d s \\
& \quad-\sum_{j=1}^{m} \sum_{i=1}^{n} \int_{t}^{T} \frac{\partial u}{\partial x_{i}}\left(s, x_{s}\right) \sigma_{i j}(s) d B_{s}^{H_{j}} \\
& =\int_{t}^{T} f\left(s, x, u\left(s, x_{s}\right), v_{1}\left(s, x_{s}\right), \ldots, v_{m}\left(s, x_{s}\right)\right) d s \\
& +\sum_{j=1}^{m} \int_{t}^{T} v_{j}\left(s, x_{s}\right) d B_{s}^{H_{j}},
\end{aligned}
$$

which is also true for $t=0$. Therefore, we have

$$
\begin{aligned}
-\int_{0}^{t}\left\{\frac{\partial u}{\partial s}\left(s, x_{s}\right)+\sum_{i=1}^{n} \frac{\partial u}{\partial x_{i}}\left(s, x_{s}\right) b_{i}(s)\right. \\
\left.\quad+\frac{1}{2} \sum_{i, j=1}^{n} \frac{\partial^{2} u}{\partial x_{i} x_{j}}\left(s, x_{s}\right) \sum_{k=1}^{m}\left[\frac{d}{d s}\left\langle\sigma_{i k}, \sigma_{j k}\right\rangle_{k, s}\right]\right\} d s \\
\quad-\sum_{j=1}^{m} \sum_{i=1}^{n} \int_{0}^{t} \frac{\partial u}{\partial x_{i}}\left(s, x_{s}\right) \sigma_{i j}(s) d B_{s}^{H_{j}} \\
=\int_{0}^{t} f\left(s, x, u\left(s, x_{s}\right), v_{1}\left(s, x_{s}\right), \ldots, v_{m}\left(s, x_{s}\right)\right) d s \\
+\sum_{j=1}^{m} \int_{0}^{t} v_{j}\left(s, x_{s}\right) d B_{s}^{H_{j}} .
\end{aligned}
$$

From Lemma 7, we get

$$
\begin{gathered}
v_{1}\left(s, x_{s}\right)=-\sum_{i=1}^{n} \sigma_{i 1}(t) u_{x_{i}}\left(t, x_{t}\right) \\
\vdots \\
v_{m}\left(s, x_{s}\right)=-\sum_{i=1}^{n} \sigma_{i m}(t) u_{x_{i}}\left(t, x_{t}\right),
\end{gathered}
$$

which completes the proof.

In order to show the existence and uniqueness of solution to (52), we introduce the following set.

$\mathscr{C}=\{\varphi(t, x) ; \varphi(t, x)$ is continuously differentiable with respect to $t$ and twice continuously differentiable with respect to $x$.

And let $\mathscr{V}_{T}$ denote the set of the process $Y_{t}$ of the form

$$
\mathscr{V}_{T}=\left\{Y .=\varphi(\cdot, x .) ; \varphi\left(t, x_{t}\right) \in \mathscr{C}, \forall t \in[0, T]\right\} .
$$


Let $\widetilde{\mathscr{V}}_{T}$ be the completion of $\mathscr{V}_{T}$ under the following $\beta$ norm:

$$
\|Y\|_{\beta}^{2}=\int_{0}^{T} e^{\beta t} \mathbb{E}\left|Y_{t}\right|^{2} d t=\int_{0}^{T} e^{\beta t} \mathbb{E}\left|\varphi\left(t, x_{t}\right)\right|^{2} d t,
$$

where $\beta$ is a positive arbitrary real-valued parameter.

We want to show that the solution to (52) in $\widetilde{\mathscr{V}}_{T}$ exists uniquely. Let $y_{t}=\varphi\left(t, x_{t}\right), z_{t}=\left(z_{1, t}, \ldots, z_{m, t}\right)=$ $\left(\psi_{1}\left(t, x_{t}\right), \ldots, \psi_{m}\left(t, x_{t}\right)\right)$ be given. Consider the following backward stochastic differential equation:

$$
\begin{aligned}
d Y_{t} & =-f\left(t, x_{t}, y_{t}, z_{t}\right) d t-\sum_{j=1}^{m} Z_{j, t} d B_{t}^{H_{j}}, \\
Y_{T} & =g\left(x_{T}\right) .
\end{aligned}
$$

Theorem 9. Assume that $\left(H_{5}\right)-\left(H_{6}\right)$ and let $y_{t}=\varphi\left(t, x_{t}\right)$ and $z_{1, t}=\psi_{1}\left(t, x_{t}\right), \ldots, z_{m, t}=\psi_{m}\left(t, x_{t}\right) \in \mathscr{C}$; then the solution $\left(Y_{t}, Z_{1, t}, \ldots, Z_{m, t}\right)$ to (69) satisfies $Y, Z_{1,}, \ldots, Z_{m, .} \in \mathscr{V}_{T}$.

Proof. By (69), we know

$$
\begin{aligned}
Y_{t}= & g\left(x_{T}\right) \\
& +\int_{t}^{T} f\left(s, x_{s}, \varphi\left(s, x_{s}\right), \psi_{1}\left(s, x_{s}\right), \ldots, \psi_{m}\left(s, x_{s}\right)\right) d s \\
& +\sum_{j=1}^{m} \int_{t}^{T} Z_{j, s} d B_{s}^{H_{j}} .
\end{aligned}
$$

Taking the quasi-conditional expectation, we have

$$
\begin{aligned}
Y_{t}=\widehat{\mathbb{E}}_{P}^{H}\left[g\left(x_{T}\right)\right. & \\
& +\int_{t}^{T} f\left(s, x_{s}, \varphi\left(s, x_{s}\right), \psi_{1}\left(s, x_{s}\right), \ldots,\right. \\
& \left.\left.\psi_{m}\left(s, x_{s}\right)\right) d s \mid \mathscr{F}_{t}\right] \\
= & P_{A_{T}-A_{t}} g\left(x_{t}\right)+\int_{t}^{T} P_{A_{T}-A_{t}} \tilde{f}\left(s, x_{s}\right) d s
\end{aligned}
$$

where $\tilde{f}\left(s, x_{s}\right)=f\left(s, x_{s}, \varphi\left(s, x_{s}\right), \psi_{1}\left(s, x_{s}\right), \ldots, \psi_{m}\left(s, x_{s}\right)\right)$. Obviously, we have $Y_{t}$ being in $\mathscr{C}$.

By $(70)$, let $t=0$; we get

$$
\begin{aligned}
Y_{0}= & g\left(x_{T}\right) \\
& +\int_{0}^{T} f\left(s, x_{s}, \varphi\left(s, x_{s}\right), \psi_{1}\left(s, x_{s}\right), \ldots, \psi_{m}\left(s, x_{s}\right)\right) d s \\
& +\sum_{j=1}^{m} \int_{0}^{T} Z_{j, s} d B_{s}^{H_{j}} .
\end{aligned}
$$

Taking the quasi-conditional expectation, we deduce

$$
\begin{aligned}
Y_{0}=\hat{\mathbb{E}}_{P}^{H}\left[\left(g\left(x_{T}\right)\right.\right. & \\
& +\int_{0}^{T} f\left(s, x_{s}, \varphi\left(s, x_{s}\right), \psi_{1}\left(s, x_{s}\right), \ldots,\right. \\
& \left.\left.\left.\psi_{m}\left(s, x_{s}\right)\right) d s\right) \mid \mathscr{F}_{t}\right] \\
+ & \sum_{j=1}^{m} \int_{0}^{t} Z_{j, s} d B_{s}^{H_{j}} .
\end{aligned}
$$

By the fractional Clark formula [20], we have

$$
\begin{aligned}
& Z_{j, t} \\
& =-\widehat{\mathbb{E}}_{P}^{H} \\
& \quad \cdot\left[\left(D_{t}^{H_{j}} g\left(x_{T}\right)+D_{t}^{H_{j}}\right.\right. \\
& \quad \cdot \int_{t}^{T} f\left(s, x_{s}, \varphi\left(s, x_{s}\right), \psi_{1}\left(s, x_{s}\right),\right. \\
& \left.\left.\left.\quad \ldots, \psi_{m}\left(s, x_{s}\right)\right) d s\right) \mid \mathscr{F}_{t}\right]
\end{aligned}
$$$$
\begin{aligned}
=-\widehat{\mathbb{E}}_{P}^{H}\left\{\sum_{i=1}^{n} \sigma_{i j}(t) \frac{\partial}{\partial x_{i}} g\left(x_{T}\right)\right. \\
+\int_{t}^{T}\left[\sum _ { i = 1 } ^ { n } \sigma _ { i j } ( t ) \frac { \partial } { \partial x _ { i } } f \left(s, x_{s}, \varphi\left(s, x_{s}\right), \psi_{1}\left(s, x_{s}\right),\right.\right.
\end{aligned}
$$$$
\left.\ldots, \psi_{m}\left(s, x_{s}\right)\right)
$$$$
+\frac{\partial}{\partial y} f\left(s, x_{s}, \varphi\left(s, x_{s}\right), \psi_{1}\left(s, x_{s}\right)\right.
$$$$
\left.\ldots, \psi_{m}\left(s, x_{s}\right)\right)
$$$$
\cdot \sum_{i=1}^{n} \sigma_{i j}(t) \varphi_{x_{i}}\left(s, x_{s}\right)
$$$$
+\sum_{j=1}^{m} \frac{\partial}{\partial z_{j}} f\left(s, x_{s}, \varphi\left(s, x_{s}\right), \psi_{1}\left(s, x_{s}\right),\right.
$$$$
\left.\ldots, \psi_{m}\left(s, x_{s}\right)\right)
$$$$
\left.\left.\cdot \sum_{i=1}^{n} \sigma_{i j}(t) \psi_{j x_{i}}\left(s, x_{s}\right)\right] d s \mid \mathscr{F}_{t}\right\}
$$ 


$$
\begin{aligned}
& =-\sum_{i=1}^{n} \sigma_{i j}(t) \widehat{\mathbb{E}}_{P}^{H} \\
& \left\{\frac{\partial}{\partial x_{i}} g\left(x_{T}\right)\right. \\
& +\int_{t}^{T}\left[\frac { \partial } { \partial x _ { i } } f \left(s, x_{s}, \varphi\left(s, x_{s}\right), \psi_{1}\left(s, x_{s}\right),\right.\right. \\
& \left.\ldots, \psi_{m}\left(s, x_{s}\right)\right) \\
& +\frac{\partial}{\partial y} f\left(s, x_{s}, \varphi\left(s, x_{s}\right),\right. \\
& \left.\psi_{1}\left(s, x_{s}\right), \ldots, \psi_{m}\left(s, x_{s}\right)\right) \\
& \text { - } \varphi_{x_{i}}\left(s, x_{s}\right) \\
& +\sum_{j=1}^{m} \frac{\partial}{\partial z_{j}} f\left(s, x_{s}, \varphi\left(s, x_{s}\right),\right. \\
& \psi_{1}\left(s, x_{s}\right), \ldots, \\
& \left.\psi_{m}\left(s, x_{s}\right)\right) \\
& \left.\left.\cdot \psi_{j x_{i}}\left(s, x_{s}\right)\right] d s \mid \mathscr{F}_{t}\right\} \\
& =-\sum_{i=1}^{n} \sigma_{i j}(t) \widehat{\mathbb{E}}_{P}^{H}\left[\frac{\partial}{\partial x_{i}} g\left(x_{T}\right) \mid \mathscr{F}_{t}\right] \\
& -\sum_{i=1}^{n} \sigma_{i j}(t) \int_{t}^{T} \widehat{\mathbb{E}}_{P}^{H}\left[\tilde{f}_{i}\left(s, x_{s}\right) \mid \mathscr{F}_{t}\right] d s,
\end{aligned}
$$

where

$$
\begin{aligned}
& \tilde{f}_{i}\left(s, x_{s}\right) \\
& =\frac{\partial}{\partial x_{i}} f\left(s, x_{s}, \varphi\left(s, x_{s}\right), \psi_{1}\left(s, x_{s}\right), \ldots, \psi_{m}\left(s, x_{s}\right)\right) \\
& \quad+\frac{\partial}{\partial y} f\left(s, x_{s}, \varphi\left(s, x_{s}\right), \psi_{1}\left(s, x_{s}\right), \ldots, \psi_{m}\left(s, x_{s}\right)\right) \\
& \quad \cdot \varphi_{x_{i}}\left(s, x_{s}\right) \\
& \quad+\sum_{j=1}^{m} \frac{\partial}{\partial z_{j}} f\left(s, x_{s}, \varphi\left(s, x_{s}\right), \psi_{1}\left(s, x_{s}\right), \ldots, \psi_{m}\left(s, x_{s}\right)\right) \\
& \quad \cdot \psi_{j x_{i}}\left(s, x_{s}\right) d s .
\end{aligned}
$$

From the expressions of $Z_{j, t}, j=1, \ldots, m$, we easily infer that $Z_{j, .} \in \mathscr{V}_{T}, j=1, \ldots, m$. We complete the proof.

Now we give the theorem of existence and uniqueness of the solution to BSDE (52).
Theorem 10. Assume that $\left(H_{1}\right)-\left(H_{6}\right)$; then BSDE (52) has a unique solution in $\mathscr{V}_{T}$.

Proof. Let $y_{t}^{i}, z_{j, t}^{i} \in \mathscr{C}, j=1, \ldots, m, i=1,2$. From Theorem 8, we know that $Y_{t}^{i}=\varphi^{i}\left(t, x_{t}\right), Z_{j, t}=\psi_{j}^{i}\left(t, x_{t}\right) \in \mathscr{C}$, $j=1, \ldots, m, i=1,2$, satisfy (70) with possibly different terminal conditions:

$$
\begin{aligned}
Y_{t}^{i}= & g^{i}\left(x_{T}\right) \\
& +\int_{t}^{T} f\left(s, x_{s}, \varphi^{i}\left(s, x_{s}\right), \psi_{1}^{i}\left(s, x_{s}\right), \ldots, \psi_{m}^{i}\left(s, x_{s}\right)\right) d s \\
& +\sum_{j=1}^{m} \int_{t}^{T} Z_{j, s}^{i} d B_{s}^{H_{j}} .
\end{aligned}
$$

Denote

$$
\begin{aligned}
& \widehat{Y}_{t}=Y_{t}^{1}-Y_{t}^{2}, \\
& \widehat{g}\left(x_{T}\right)=g^{1}\left(x_{T}\right)-g^{2}\left(x_{T}\right), \\
& \widehat{Z}_{j, t}=Z_{j, t}^{1}-Z_{j, t}^{2}, \quad j=1, \ldots, m, \\
& \widehat{f}_{t} \\
& \quad=f\left(s, x_{s}, \varphi^{1}\left(s, x_{s}\right), \psi_{1}^{1}\left(s, x_{s}\right), \ldots, \psi_{m}^{1}\left(s, x_{s}\right)\right) \\
& \quad-f\left(s, x_{s}, \varphi^{2}\left(s, x_{s}\right), \psi_{1}^{2}\left(s, x_{s}\right), \ldots, \psi_{m}^{2}\left(s, x_{s}\right)\right) .
\end{aligned}
$$

Then we get

$$
d \widehat{Y}_{t}=-\widehat{f}_{t} d t-\sum_{j=1}^{m} \widehat{Z}_{j, t} d B_{t}^{H_{j}}
$$

and we deduce

$$
d \widehat{Y}_{t}^{2}=-2 \widehat{Y}_{t} \widehat{f}_{t} d t-2 \widehat{Y}_{t} \sum_{j=1}^{m} \widehat{Z}_{j, t} d B_{t}^{H_{j}}-2 \sum_{j=1}^{m} \widehat{Z}_{j, t} \mathbb{D}_{t}^{H_{j}} \widehat{Y}_{t} d t
$$

However, if $Y_{t}^{1}=\tilde{\varphi}\left(t, x_{t}\right)$, by Theorem 8 , we have

$$
Z_{j, t}^{1}=-\sum_{i=1}^{n} \sigma_{i j}(t) \widetilde{\varphi}_{x_{i}}\left(t, x_{t}\right), \quad j=1, \ldots, m .
$$

By the property of Malliavin derivative relative to the fractional Brownian motion, we obtain

$$
\begin{aligned}
\mathbb{D}_{t}^{H_{j}} Y_{t}^{1} & =\sum_{i=1}^{n} \widetilde{\varphi}_{x_{i}}\left(t, x_{t}\right) \int_{0}^{t} \phi_{j}(t, s) D_{s}^{H_{j}} x_{i}(t) d s \\
& =\sum_{i=1}^{n} \widetilde{\varphi}_{x_{i}}\left(t, x_{t}\right) \int_{0}^{t} \phi_{j}(t, s) \sigma_{i j}(s) d s \\
& =-\sum_{i=1}^{n} \frac{\widehat{\sigma}_{i j}(t)}{\sigma_{i j}(t)} Z_{j, t}^{1} .
\end{aligned}
$$


Moreover, by (79) and (81), we get

$$
\begin{aligned}
d \widehat{Y}_{t}^{2}= & -2 \widehat{Y}_{t} \widehat{f}_{t} d t-2 \widehat{Y}_{t} \sum_{j=1}^{m} \widehat{Z}_{j, t} d B_{t}^{H_{j}} \\
& +2 \sum_{j=1}^{m} \sum_{i=1}^{n} \frac{\widehat{\sigma}_{i j}(t)}{\sigma_{i j}(t)} \widehat{Z}_{j, t}^{2} d t .
\end{aligned}
$$

By (19), we have

$$
\begin{aligned}
d\left(e^{\beta t} \widehat{Y}_{t}^{2}\right)= & \beta e^{\beta t} \widehat{Y}_{t}^{2} d t-2 e^{\beta t} \widehat{Y}_{t} \widehat{f}_{t}-2 e^{\beta t} \widehat{Y}_{t} \sum_{j=1}^{m} \widehat{Z}_{j, t} d B_{t}^{H_{j}} \\
& +2 e^{\beta t} \sum_{j=1}^{m} \sum_{i=1}^{n} \frac{\widehat{\sigma}_{i j}(t)}{\sigma_{i j}(t)} \widehat{Z}_{j, t}^{2} d t
\end{aligned}
$$

Taking the expectations and integrating on both sides of (83), we obtain

$$
\begin{array}{rl}
e^{\beta t} & \mathbb{E} \widehat{Y}_{t}^{2}+2 \int_{t}^{T} e^{\beta s} \sum_{j=1}^{m} \mathbb{E}\left(\sum_{i=1}^{n} \frac{\widehat{\sigma}_{i j}(s)}{\sigma_{i j}(s)} \widehat{Z}_{j, s}^{2}\right) d s \\
= & e^{\beta T} \mathbb{E} \widehat{Y}_{T}^{2}-\beta \int_{t}^{T} e^{\beta s} \mathbb{E} \widehat{Y}_{s}^{2} d s+2 \int_{t}^{T} e^{\beta t} \mathbb{E}\left(\widehat{Y}_{s} \widehat{f}_{s}\right) d s .
\end{array}
$$

From (51) and (84), we have

$$
\begin{aligned}
e^{\beta t} \mathbb{E} \widehat{Y}_{t}^{2}+2 n c_{0} \sum_{j=1}^{m} \int_{t}^{T} e^{\beta s} \mathbb{E} \widehat{Z}_{j, s}^{2} d s \\
\leq e^{\beta T} \mathbb{E} \widehat{Y}_{T}^{2}-\beta \int_{t}^{T} e^{\beta s} \mathbb{E} \widehat{Y}_{s}^{2} d s+2 \int_{t}^{T} e^{\beta t} \mathbb{E}\left(\widehat{Y}_{s} \widehat{f}_{s}\right) d s \\
\leq e^{\beta T} \mathbb{E} \widehat{Y}_{T}^{2}-\beta \int_{t}^{T} e^{\beta s} \mathbb{E}\left[\widehat{Y}_{s}-\frac{\widehat{f}_{s}}{\beta}\right]^{2} d s \\
\quad+\frac{1}{\beta} \int_{t}^{T} e^{\beta t} \mathbb{E} \widehat{f}_{s}^{2} d s \leq e^{\beta T} \mathbb{E} \widehat{Y}_{T}^{2}+\frac{1}{\beta} \int_{t}^{T} e^{\beta t} \mathbb{E} \widehat{f}_{s}^{2} d s .
\end{aligned}
$$

By (85), we get

$$
\begin{aligned}
e^{\beta t} \mathbb{E} \widehat{Y}_{t}^{2} \leq & e^{\beta T} \mathbb{E} \widehat{Y}_{T}^{2}+\frac{1}{\beta} \int_{0}^{T} e^{\beta t} \mathbb{E} \widehat{f}_{s}^{2} d s \\
\sum_{j=1}^{m} \int_{0}^{T} e^{\beta s} \mathbb{E} \widehat{Z}_{j, s}^{2} d s \leq & \frac{1}{2 n c_{0}} e^{\beta T} \mathbb{E} \widehat{Y}_{T}^{2} \\
& +\frac{1}{2 n c_{0} \beta} \int_{0}^{T} e^{\beta t} \mathbb{E} \widehat{f}_{s}^{2} d s .
\end{aligned}
$$

Integrating from 0 to $T$ on both sides of (86)

$$
\int_{0}^{T} e^{\beta s} \mathbb{E} \widehat{Y}_{s}^{2} d s \leq T e^{\beta T} \mathbb{E} \widehat{Y}_{T}^{2}+\frac{T}{\beta} \int_{0}^{T} e^{\beta t} \mathbb{E} \widehat{f}_{s}^{2} d s .
$$

By (87) and (88), we derive

$$
\begin{aligned}
\|\widehat{Y}\|_{\beta}^{2}+\sum_{j=1}^{m}\left\|\widehat{Z}_{j}\right\|_{\beta}^{2} \leq & \left(\frac{e^{\beta T}}{2 n c_{0}}+T e^{\beta T}\right) \mathbb{E}\left|\widehat{Y}_{T}\right|^{2} \\
& +\frac{1}{\beta}\left(T+\frac{1}{2 n c_{0}}\right)\|\hat{f}\|_{\beta}^{2} .
\end{aligned}
$$

By the hypotheses of Theorem 10, we know that $f(t, x, y, z)$ is uniformly globally Lipschitzian with respect to $y, z$, and $\widehat{Y}_{T}=0$, so we have

$$
\begin{aligned}
& \|\widehat{Y}\|_{\beta}^{2}+\sum_{j=1}^{m}\left\|\widehat{Z}_{j}\right\|_{\beta}^{2} \\
& \quad \leq \frac{L}{\beta}\left(T+\frac{1}{2 n c_{0}}\right)\left[\|\hat{y}\|_{\beta}^{2}+\sum_{j=1}^{m}\left\|\widehat{z}_{j}\right\|_{\beta}^{2}\right],
\end{aligned}
$$

where $L$ is Lipschitzian constant of the function $f$.

Choosing $\beta$ sufficiently large, we see that the mapping $\left(y, z_{1}, \ldots, z_{m}\right) \rightarrow\left(Y, Z_{1}, \ldots, Z_{m}\right)$ determined by $(70)$ is a contraction in $\widetilde{\mathscr{V}}_{T}$. Using the fixed point principle, we deduce that the solution to (52) exists uniquely, and the proof is completed.

\section{Linear Backward Stochastic Differential Equations}

In this section we discuss the following linear backward stochastic differential equation:

$$
\begin{aligned}
& d y_{t}=-\left[\alpha_{t}+\beta_{t} y_{t}+\sum_{i=1}^{m} \gamma_{i, t} z_{i, t}\right] d t-\sum_{i=1}^{m} z_{i, t} d B_{t}^{H_{i}}, \\
& y_{T}=\xi
\end{aligned}
$$

where $\alpha_{t}, \beta_{t}$, and $\gamma_{i, t}, i=1, \ldots, m$, are given, continuous, and adapted processes and $\xi \in \mathscr{F}_{T}$; in particular we allow the terminal condition $\xi$ to be general form. When the coefficients are deterministic and $i=1$, the equation is studied in [10-12]. When the coefficients are deterministic, $i=2$ and $H_{1}=1 / 2$, the equation is studied in [15]. This paper further studies more general linear backward stochastic differential equation. Let

$$
\rho_{t}=\exp \left\{\int_{0}^{T} \beta_{s} d s\right\}
$$

that is,

$$
d \rho_{t}=\beta_{t} \rho_{t} d t
$$


From fractional Itô chain rule (18), we have

$$
\begin{aligned}
d\left(\rho_{t} y_{t}\right)= & \rho_{t} d y_{t}+y_{t} d \rho_{t}-\sum_{i=1}^{m} z_{i, t} \mathbb{D}_{t}^{H_{i}} \rho_{t} d t \\
& -\left[\alpha_{t} \rho_{t}+\sum_{i=1}^{m} \gamma_{i, t} \rho_{t} z_{i, t}+\sum_{i=1}^{m} z_{i, t} \mathbb{D}_{t}^{H_{i}} \rho_{t}\right] d t \\
& -\sum_{i=1}^{m} z_{i, t} \rho_{t} d B_{t}^{H_{i}} \\
= & -\alpha_{t} \rho_{t} \\
& -\sum_{i=1}^{m} \rho_{t} z_{i, t}\left[\left(\gamma_{i, t}+\frac{\mathbb{D}_{t}^{H_{i}} \rho_{t}}{\rho_{t}}\right) d t+d B_{t}^{H_{i}}\right] \\
= & -\alpha_{t} \rho_{t}-\sum_{i=1}^{m} \rho_{t} z_{i, t} d \widehat{B}_{t}^{H_{i}},
\end{aligned}
$$

where

$$
\begin{aligned}
d \widehat{B}_{t}^{H_{i}} & =\left(\gamma_{i, t}+\frac{\mathbb{D}_{t}^{H_{i}} \rho_{t}}{\rho_{t}}\right) d t+d B_{t}^{H_{i}} \\
& =d B_{t}^{H_{i}}+\left(\gamma_{i, t}+\int_{0}^{T} \mathbb{D}_{s}^{H_{i}} \beta_{s} d s\right) d t
\end{aligned}
$$

which is a fractional Brownian motion under the new probability measure $Q$ defined by

$$
\begin{aligned}
\frac{d Q}{d P}=\exp \left\{\sum _ { i = 1 } ^ { m } \left(\int_{0}^{T}\left(\mathscr{K}_{H_{i}}^{-1} \int_{0}^{\cdot} b_{i, r} d r\right)(s) d W_{s}\right.\right. \\
\left.\left.\quad-\frac{1}{2} \int_{0}^{T}\left(\mathscr{K}_{H_{i}}^{-1} \int_{0} b_{i, r} d r\right)^{2}(s) d s\right)\right\},
\end{aligned}
$$

where $b_{i, r}=\gamma_{i, r}+\int_{0}^{r} \mathbb{D}_{r}^{H_{i}} \beta_{s} d s$ and $\mathscr{K}_{H_{i}}^{-1}(t)$ is defined in (23). From the definitions of the probability measures $Q$ in (96), according to the Girsanov theorem, we know that $\widehat{B}_{t}^{H_{i}}, i=1, \ldots, m$, is a fractional Brownian motion relative to probability $Q$. Thus we have

$$
\rho_{t} y_{t}=\rho_{T} \xi+\int_{0}^{T} \alpha_{s} \rho_{s} d s+\sum_{i=1}^{m} \int_{0}^{T} \rho_{t} z_{i, s} d \widehat{B}_{s}^{H_{i}} .
$$

Therefore

$$
\rho_{t} y_{t}=\widehat{\mathbb{E}}_{\mathrm{Q}}^{H}\left[\rho_{T} \xi+\int_{t}^{T} \alpha_{s} \rho_{s} d s \mid \mathscr{F}_{t}\right]
$$

that is,

$$
y_{t}=\rho_{t}^{-1} \widehat{\mathbb{E}}_{\mathrm{Q}}^{H}\left[\rho_{T} \xi+\int_{t}^{T} \alpha_{s} \rho_{s} d s \mid \mathscr{F}_{t}\right]
$$

From above deduction, we have the following theorem.
Theorem 11. Let $\alpha_{t}, \beta_{t}$, and $\gamma_{i, t}, i=1, \ldots, m$, be given, continuous, and adapted processes and $\xi \in \mathscr{F}_{T}$. Suppose

$$
\mathbb{E}\left[\exp \left\{\frac{1}{2} \sum_{i=1}^{m} \int_{0}^{T}\left(\mathscr{K}_{H_{i}}^{-1} \int_{0}^{\cdot} b_{i, r} d r\right)^{2}(s) d s\right\}\right]<\infty,
$$

where $b_{i, r}=\gamma_{i, r}+\int_{0}^{r} \mathbb{D}_{r}^{H_{i}} \beta_{s} d s$. Then the solution to (91) exists uniquely. Moreover, we have

$$
y_{t}=\rho_{t}^{-1} \widehat{\mathbb{E}}_{\mathrm{Q}}^{H}\left[\rho_{T} \xi+\int_{t}^{T} \alpha_{s} \rho_{s} d s \mid \mathscr{F}_{t}\right] .
$$

Proof. The existence and uniqueness for $y_{t}$ can be obtained from the above argument. For the existence and uniqueness of $z_{i, t}, i=1, \ldots, m$, we apply Theorem 12.1 of [21]. We complete the proof of the theorem.

\section{Conclusion}

We study the solutions to the backward stochastic differential equations driven by multidimensional fractional Brownian motions, with a more general terminal condition $y_{T}=$ $g\left(x_{1}(T), \ldots, x_{n}(T)\right)$, where $x_{i}(t)=x_{i}(0)+\int_{0}^{t} b_{i}(s) d s+$ $\sum_{j=1}^{m} \int_{0}^{t} \sigma_{i j}(s) d B_{s}^{H_{j}}, i=1, \ldots, n$. Using the quasi-conditional expectation and multidimensional fractional Itô formula, we obtain the existence and uniqueness of the solutions to BSDEs, where a fixed point principle is employed. Finally, solutions to linear backward stochastic differential equations driven by multidimensional fractional Brownian motions are investigated. Thus we extend some results of [10-12, 15]. Nowadays the theory and applications of fractional stochastic differential equations play a crucial role in characterizing many social, physical, biological, and engineering problems. In the future, we may extend our work to integrodifferential inclusions [22] and fractional integrodifferential inclusions [23].

\section{Conflict of Interests}

The authors declare that there is no conflict of interests regarding the publication of this paper.

\section{Acknowledgments}

The authors thank the referees for careful reading and their helpful comments and suggestions for the improvement of this paper. This research is partially supported by the National Natural Science Foundations of China under Grants numbers 91130003 and 11171189.

\section{References}

[1] A. N. Kolmogorov, "Wienersche Spiralen und einige andere interessante Kurven im Hilbertschen Raum," Comptes Rendus (Doklady) de l'Académie des Sciences de l'URSS, vol. 26, pp. 115118, 1940.

[2] B. B. Mandelbrot and J. W. van Ness, "Fractional Brownian motions, fractional noises and applications," SIAM Review, vol. 10, pp. 422-437, 1968. 
[3] T. E. Duncan, Y. Hu, and B. Pasik-Duncan, "Stochastic calculus for fractional Brownian motion. I. Theory," SIAM Journal on Control and Optimization, vol. 38, no. 2, pp. 582-612, 2000.

[4] Y. S. Mishura, Stochastic Calculus for Fractional Brownian Motion and Related Processes, Springer, Berlin, Germany, 2008.

[5] P. Carmona, L. Coutin, and G. Montseny, "Stochastic integration with respect to fractional Brownian motion," Annales de l'Institut Henri Poincare (B) Probability and Statistics, vol. 39, no. 1, pp. 27-68, 2003.

[6] L. Coutin and Z. Qian, "Stochastic differential equations for fractional Brownian motions," Comptes Rendus de l'Académie des Sciences Series I: Mathematics, vol. 331, no. 1, pp. 75-80, 2000.

[7] W. Xiao, W. Zhang, W. Xu, and X. Zhang, "The valuation of equity warrants in a fractional Brownian environment," Physica A. Statistical Mechanics and Its Applications, vol. 391, no. 4, pp. 1742-1752, 2012.

[8] S. Rostek and R. Schöbel, "A note on the use of fractional Brownian motion for financial modeling," Economic Modelling, vol. 30, no. 1, pp. 30-35, 2013.

[9] E. Pardoux and S. G. Peng, "Adapted solution of a backward stochastic differential equation," Systems \& Control Letters, vol. 14, no. 1, pp. 55-61, 1990.

[10] Y. Hu and S. Peng, "Backward stochastic differential equation driven by fractional Brownian motion," SIAM Journal on Control and Optimization, vol. 48, no. 3, pp. 1675-1700, 2009.

[11] C. Bender, "Explicit solutions of a class of linear fractional BSDEs," Systems \& Control Letters, vol. 54, no. 7, pp. 671-680, 2005.

[12] H. Zhang, "Properties of solution of fractional backward stochastic differential equation," Applied Mathematics and Computation, vol. 228, pp. 446-453, 2014.

[13] L. Maticiuc and T. Nie, "Fractional backward stochastic differential equations and fractional backward variational inequalities," Journal of Theoretical Probability, 2013.

[14] K. Jańczak-Borkowska, "Generalized BSDEs driven by fractional Brownian motion," Statistics and Probability Letters, vol. 83, no. 3, pp. 805-811, 2013.

[15] W. Fei, D.-F. Xia, and S. Zhang, "Solutions to BSDEs driven by both standard and fractional Brownian motions," Acta Mathematicae Applicatae Sinica, vol. 29, no. 2, pp. 329-354, 2013.

[16] F. Biagini, B. Øksendal, A. Sulem, and N. Wallner, "An introduction to white-noise theory and Malliavin calculus for fractional Brownian motion," Proceedings of the Royal Society of London A, vol. 460, no. 2041, pp. 347-372, 2004.

[17] W. Dai and C. C. Heyde, "Itô's formula with respect to fractional Brownian motion and its application," Journal of Applied Mathematics and Stochastic Analysis, vol. 9, no. 4, pp. 439-448, 1996.

[18] D. Revuz and M. Yor, Continuous Martingales and Brownian Motion, Springer, Berlin, Germany, 1999.

[19] Y. Hu, D. Ocone, and J. Song, "Some results on backward stochastic differential equation driven by fractional Brownian motions," in Stochastic Analysis and Applications to Finance, pp. 225-242, World Scientific Publishing Company, Hackensack, NJ, USA, 2012.

[20] Y. Hu, "Integral tranformations and anticipative calculus for fractional Brownian motions," Memoirs of the American Mathematical Society, vol. 175, no. 825, 2005.

[21] D. Nualart, The Malliavin Calculus and Related Topics, Springer, New York, NY, USA, 2nd edition, 2006.

[22] P. Balasubramaniam and D. Vinayagam, "Existence of solutions of nonlinear stochastic integrodifferential inclusions in a
Hilbert space," Computers \& Mathematics with Applications, vol. 50, no. 5-6, pp. 809-821, 2005.

[23] P. Balasubramaniam and P. Tamilalagan, "Approximate controllability of a class of fractional neutral stochastic integrodifferential inclusions with infinite delay by using Mainardi's function," Applied Mathematics and Computation, vol. 256, pp. 232-246, 2015. 


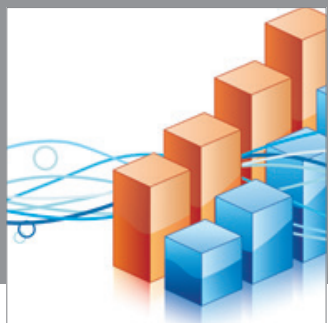

Advances in

Operations Research

mansans

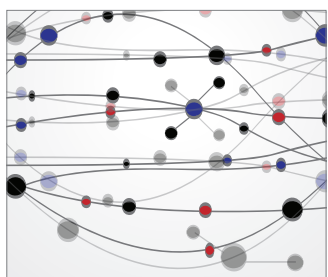

The Scientific World Journal
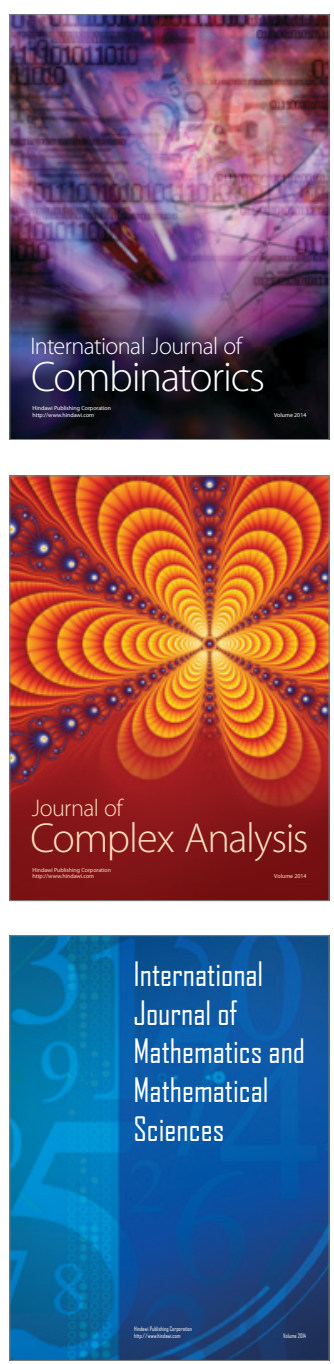
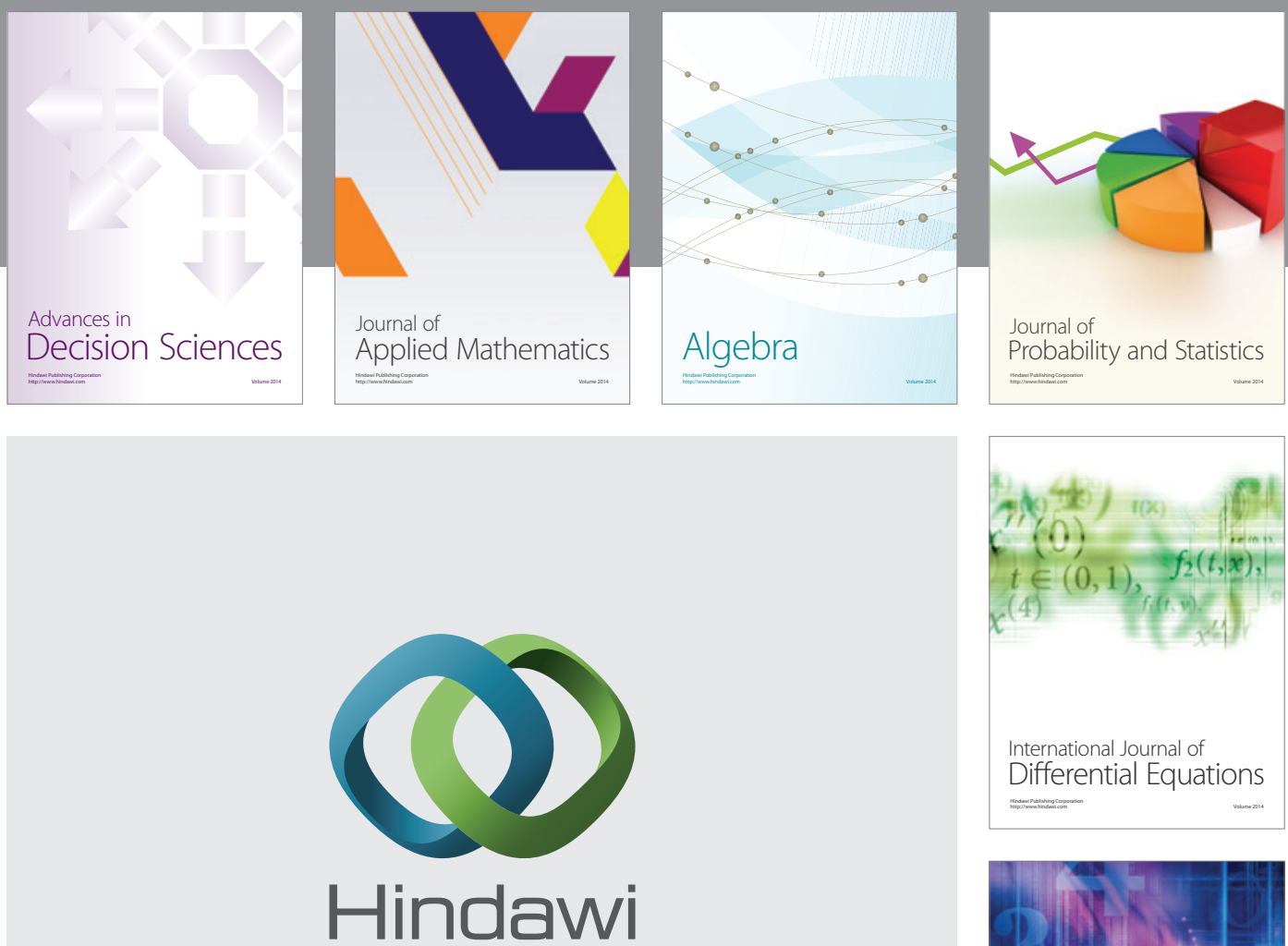

Submit your manuscripts at http://www.hindawi.com
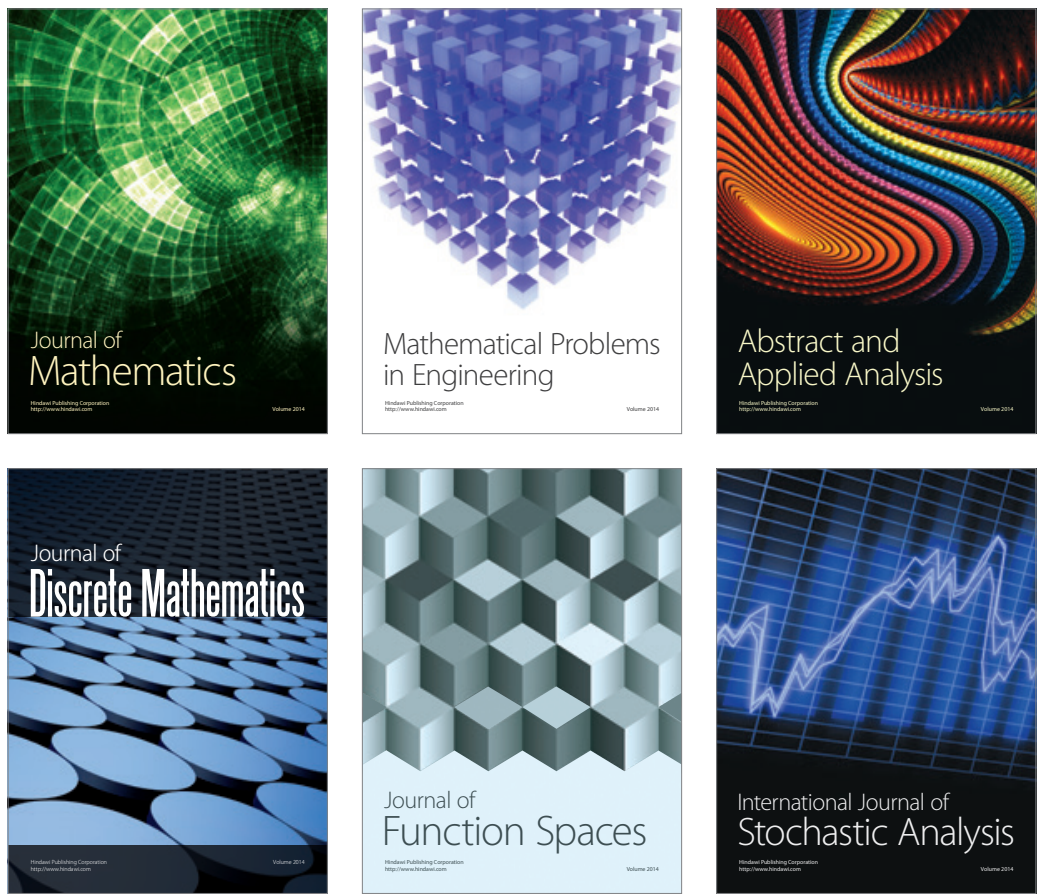

Journal of

Function Spaces

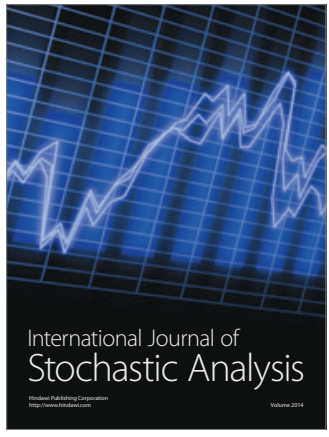

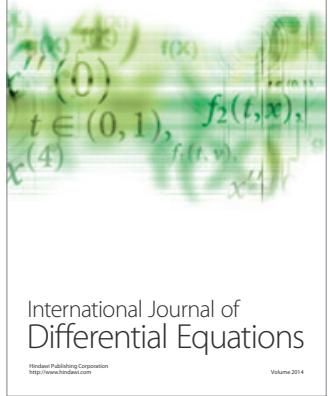
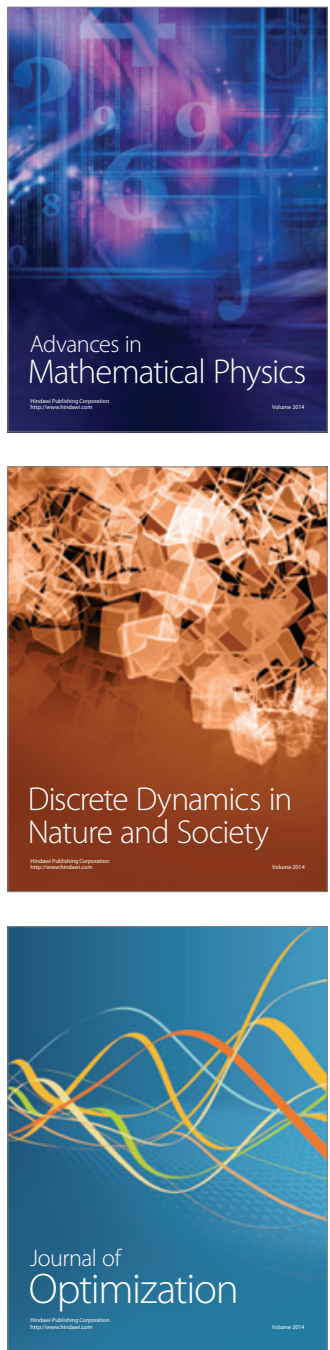